\title{
Dark Energy is Dark Matter below Absolute Zero in Black Holes
}

\author{
$\underline{\text { Roumen Tsekov }}$ \\ Department of Physical Chemistry, University of Sofia, 1164 Sofia, Bulgaria
}

\begin{abstract}
It is proven that the Clapeyron-Mendeleev equation, originally discovered for gases with large entropy, describes the state of dark matter and energy due to enormous self-gravitation. It is shown that the dark matter exists as compact cold neutrino stars at positive temperature with size twice the Schwarzschild radius. In contrast, the dark energy is dark matter at temperature below the absolute zero, which is locked entirely in black holes, where the pressure is negative as required for the Universe intrinsic expansion. The derived dark phase diagram predicts negative critical temperatures for the Big Bang and the Big Rip of black holes.
\end{abstract}

Keywords: Neutrino, Dark Matter, Dark Energy, Black Holes, Negative Temperature

According to modern understanding, the Universe contains four forms of matter. The ordinary matter, built up from electrons and protons, amounts solely $5 \%$ from the Universe massenergy including $0.5 \%$ electromagnetic radiation as well. The rest of the Universe consists of uncharged dark ingredients. ${ }^{1}$ Dark matter is a hypothetical substance ${ }^{2}$ which is introduced to explain several gravitational anomalies. Though it is estimated to be $27 \%$ from the Universe mass, the dark matter is not experimentally detected yet. This is theoretically attributed to inability of the dark matter to interact except gravitationally with baryons and radiation, which is typical for neutrinos. ${ }^{3}$ The latter pass undetected through ordinary matter and are proper candidates for building particles of the dark matter. ${ }^{4}$ Dark energy is required to explain the cosmic inflation. ${ }^{5}$ The intrinsic expansion of the Universe requires for the Friedmann equations a negative cosmological pressure $p=w \rho c^{2}$, where $\rho$ is mass density, $c$ is the speed of light in vacuum, and the dark energy is defined by the weird inequality $w<0$. According to the quintessence, ${ }^{1} w=L / H$ equals to the ratio between the Lagrangian $L$ and the Hamiltonian $H$ of a scalar field. So, the kinetically dominated stiff matter has $w=1$, the potentially dominated static dark energy has $w=-1$, while the so-called phantom energy possesses $w<-1$. Obviously, the Universe is rich of dark energy, being the remaining $68 \%$ from the Universe one, but it has never been observed as well. Its negative pressure hints, however, the natural hypothesis that the dark energy is entirely locked in black holes, which are sucking continuously matter and space around. Hence, the dark energy is hidden from us forever and to discover its nature, one must have a look inside a black hole, which is possible via theory only. 
According to statistical thermodynamics, the pressure can be expressed most generally by the following equation of state $p V=k_{B} T \ln \Xi$, where $V$ is volume and $T$ is temperature. The grand canonical partition function $\Xi\left(V, T,\left\{z_{k}\right\}\right)$ depends also on the fugacity $z_{k}$ of each component and the corresponding number of particles can be calculated via $N_{k}=z_{k}\left(\partial_{z_{k}} \ln \Xi\right)_{V, T}$. In dilute gases at high temperature the large entropy drives fugacity to tend to zero. In this case, expanding $\ln \Xi$ in fugacity series around $z_{k}=0$ and keeping the linear terms only yields the relation $\ln \Xi=\sum N_{k}=N$, where $N$ is the total number of particles. Thus, one arrives to the famous Clapeyron-Mendeleev equation of state

$$
p V=N k_{B} T
$$

The derivation above confirms the Boyle-Mariotte law that any real gas tends to the ideal one at vanishing pressure. If the system is not dilute, the equation of state accounts via $\ln \Xi$ for many interparticle interactions and quantum effects, which explain the diversity of ordinary matter. On the other hand, the dark matter and energy are exposed to huge self-gravitational forces, which are attractive and cumulative. Magnified additionally by low temperature, the latter push fugacity to tend to zero again, but now $z_{k} \rightarrow 0$ is due to the enormous self-gravitation, not to the large entropy as in the ideal gas. Thus, the Clapeyron-Mendeleev equation (1) appears amazingly to be the equation of state of the dark matter and energy as well. This is not surprising, however, since in contrast to other fundamental interactions the mass increase persistently decreases the chemical potentials of all components. The other fundamental and quantum interactions become negligible compared the boundless gravity, which can lead even to collapse to black holes. For example, the gravitation causes nucleus to capture their orbital electrons in atoms of ordinary matter. Thus, the white dwarfs convert to neutron stars before the latter transform to black holes.

The conclusion above is supported too by the linear dependence of the cosmological pressure $p=w \rho c^{2}$ on the mass density. Substituting it in Eq. (1) unveils the mysterious cosmological factor $w=T$ / $\theta$ as dimensionless temperature with the characteristic temperature $\theta \equiv \mu c^{2} / k_{B}$, reflecting the average mass $\mu \equiv M / N$ per particle. In the physical cosmology, ${ }^{6} w$ is of the order of one, which suggests that the dark matter temperature is proportional to $\theta$. It is clear now that $\mu$ must be extremely small to compensate the huge value of the speed of light and to ensure the low temperature of the Universe. Looking for stable particles in the Standard Model, ${ }^{7}$ one recognizes that the cold dark side of the Universe is made of neutrinos, because their thermal velocity at low temperature is commensurable with the speed of light due to the neutrino tiny mass. The latest estimates ${ }^{8}$ for the upper limit of the neutrino mass show that the corresponding characteristic temperature is below $100 \mathrm{~K}$, which is reasonable. In contrast, the characteristic temperature for massive particles is huge, e.g. $10^{13} \mathrm{~K}$ for neutrons, being typical for hot neutron stars. 
Assuming equilibrium between dark matter and free neutrinos, one can estimate $\theta=2 \mathrm{~K}$ from the temperature of the cosmic neutrino background. ${ }^{1}$ The corresponding neutrino mass in dark matter $\mu=0.2 \mathrm{meV} / \mathrm{c}^{2}$ is far below the upper limit for free neutrino mass, but one should take into account also a significant mass defect inside dark matter, caused by the enormous self-gravitation. Amazingly, $w<0$ suggests that the dark energy represents neutrinos at temperature below absolute zero. Traditionally, temperature of gases is associated with the kinetic energy of the gas particles, which is always positive. The truth is, however, vice versa: the mean kinetic energy of gas particles is proportional to positive temperature, but temperature is a more general parameter. ${ }^{9}$ It is so important that temperature has been introduced afterwards in thermodynamics via the Zeroth Law. Since the phenomenological Third Law of thermodynamics bans temperatures below absolute zero, the dark energy must be concealed somewhere, inaccessible for our measurements. This conclusion correlates well with our leading hypothesis that the dark energy is hidden inside black holes. Evidently, neutrons break up to neutrinos during the self-gravitational collapse of neutron stars to black holes.

A free ideal gas of neutrinos, as the cosmic neutrino background, cannot explain the gravitational effect of the dark matter. Although the tiny mass, a very large number of neutrinos can form a macroscopic body, solely via self-gravitation. ${ }^{10}$ According to the general relativity theory such a spherical body is described by the Tolman-Oppenheimer-Volkoff equation ${ }^{11,12}$

$$
\partial_{r} p=-G\left(\rho+p / c^{2}\right)\left(m_{r}+4 \pi r^{3} p / c^{2}\right) / r^{2}\left(1-2 G m_{r} / c^{2} r\right) \quad \partial_{r} m_{r}=4 \pi r^{2} \rho
$$

where $m_{r}$ is the mass enclosed inside a sphere with radius $r$ and $G$ is the universal gravitational constant. Introducing here the cosmological pressure $p=w \rho c^{2}$ yields at constant temperature $w$ a complicated nonlinear differential equation for the mass density, which possesses, however, a very simple solution: ${ }^{13} \rho=M / 4 \pi R r^{2}$ or $m_{r}=M r / R$. The mass $M$ and radius $R$ of the body are related via the expression, following directly from Eq. (2), ${ }^{10}$

$$
R / r_{s}=1+(1+w)^{2} / 4 w
$$

where the body mass is hidden in the Schwarzschild radius $r_{s} \equiv 2 G M / c^{2}$. Remarkably the specific radius per unit mass $R / M$ depends solely on temperature. Therefore, Eq. (3) describes the dark phase diagram, which is relating the size, mass, and temperature of the compact body. Interestingly, the important inversion of $w$ to the reciprocal temperature $1 / w$ does not affect $R / r_{s}$. 
Let us consider the nonlinear plot of Eq. (3) in Fig. 1. As is seen, there are no black holes at positive temperature, because $R \geq 2 r_{s}$ independently from the mass $M$. Thus, the self-gravitating neutrinos form stars with radii at least twice larger than the Schwarzschild radius. The most compact neutrino stars exist at temperature $T=\theta$ and their radii $R=2 r_{s}$ correspond to the circular marginally bound orbits. Obviously, the most compact neutrino stars are cold and dark since they cannot shrink anymore to emit energy. Hence, they are proper structural elements of the dark matter, whose pressure $p=\rho c^{2}$ equals exactly to the energy density, calculated from the Einstein mass-energy relation. The dark energy possesses negative pressure $p<0$ by definition, which according to Eq. (1) means that it consists of neutrinos at negative temperature $T<0$. To see, where dark energy is, let us look for negative temperatures in Fig. (1). As is seen, the dark energy exists only in black holes, because $R \leq r_{s}$. On the other hand, the black holes contain only dark energy, which represents dark matter at temperature below absolute zero. At negative temperature particles occupy higher energy levels. ${ }^{9}$ Thus, in contrast to the dark matter, which stabilizes at the minimal specific radius $R / r_{s}=2$ for positive temperature, the dark energy favors the maximal specific radius $R / r_{s}=1$ at negative temperature. Notably, the plot in Fig. 1 possesses inversion through the point at absolute zero and the radius $3 r_{s} / 2$ of the photon sphere.

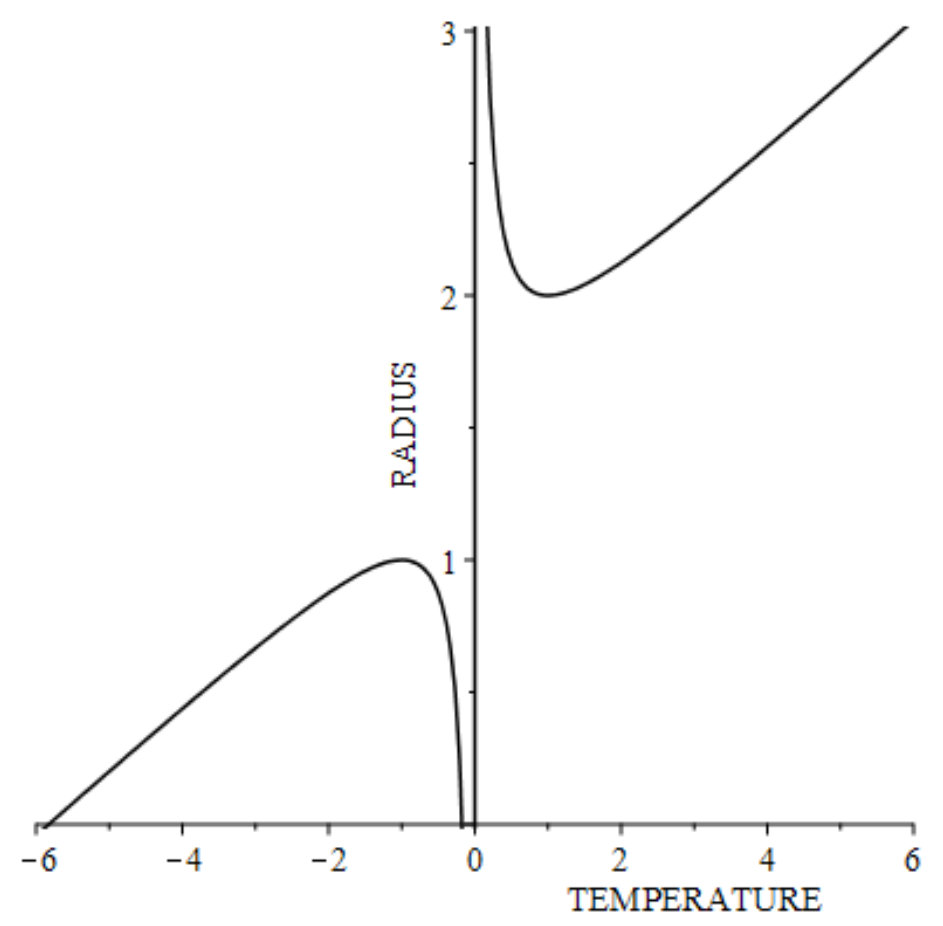

Fig. 1. The dimensionless radius $R / r_{s}$ as a function of the dimensionless temperature $w=T / \theta$

The experimental observations of the Universe expansion report that the dimensionless factor is close to minus one, indeed, and $w=-1$ agrees with the Einstein cosmological constant 
as well. ${ }^{5}$ Hence, the black holes are filled up to the Schwarzschild radius by neutrinos at temperature $T=-\theta$. The negative pressure opposes the gravitational singularity at the black hole surface. Thus, the local mass density in black holes, depending solely on temperature, is simply twice larger than $\rho$ in the dark matter stars. Obviously, the dark matter-energy phase transition occurs in the quintessence region $-1 \leq w \leq 1$ and it is always accompanied by a big freeze because temperature crosses absolute zero. The plot in Fig. 1 shows presence of two peculiar points at negative temperature. The higher $w_{B}=-3+2 \sqrt{2}$ must be the Big Bang temperature. Heating a black hole, its specific radius becomes zero at $w_{B}$, which corresponds to infinite gravitational energy. At this slightly negative temperature the black hole can burst further to generate matter with positive temperature. The lower critical temperature $w_{R}=-3-2 \sqrt{2}$ indicates the Big Rip in the phantom energy region. ${ }^{14}$ Obviously, black holes disappear at temperature below $w_{R} \cdot{ }^{15}$ Remarkably, the two critical temperatures are related via the seesaw construction $w_{R}=1 / w_{B}$.

\section{References}

1. S. Carroll, Dark Matter, Dark Energy: The Dark Side of the Universe, Teaching, Chantilly, 2007

2. V. Trimble, Existence and nature of dark matter in the Universe, Ann. Rev. Astron. Astrophys. 25 (1987) 425

3. F. Close, Neutrinos, Oxford University Press, Oxford, 2010

4. A. Merle, Sterile Neutrino Dark Matter, IOP, Bristol, 2017

5. P.J.E. Peebles and B. Ratra, The cosmological constant and dark energy, Rev. Mod. Phys. 75 (2003) 559

6. L. Bergström and A. Goobar, Cosmology and Particle Astrophysics, Springer, New York, 2006

7. R. Mann, An Introduction to the Standard Model of Particle Physics, CRC, Boca Raton, 2009

8. J.A. Formaggio, A.L.C. de Gouvea and R.G.H. Robertson, Direct measurements of neutrino mass, Phys. Rep. 914 (2021) 1

9. M. Baldovin, S. Iubini, R. Livi and A. Vulpiani, Statistical mechanics of systems with negative temperature, Phys. Rep. 923 (2021) 1

10. R. Tsekov, From black holes to neutrino stars, Ann. Univ. Sofia Fac. Phys. 112 (2020) 000

11. R.C. Tolman, Static solutions of Einstein's field equations for spheres of fluid, Phys. Rev. 55 (1939) 364

12. J.R. Oppenheimer and G.M. Volkoff, On massive neutron cores, Phys. Rev. 55 (1939) 374

13. S. Weinberg, Gravitation and Cosmology: Principles and Applications of the General Theory of Relativity, Wiley, New York, 2013

14. R.R. Caldwell, M. Kamionkowski and N.N. Weinberg, Phantom energy: Dark energy with $w<-1$ causes a cosmic doomsday, Phys. Rev. Lett. 91 (2003) 071301

15. K. Mack, The End of Everything (Astrophysically Speaking), Scribner, New York, 2021 